\title{
NSAIDS, malignancies and GI adverse events: a FAERS analysis
}

\author{
Florent Richy* \\ Director Bluepoint Consulting SCS, Liege / Consultant professor University of Liege, Belgium
}

\begin{abstract}
Non-steroidal anti-inflammatory drugs (NSAIDs) are amongst the most widely used drugs worldwide to treat conditions were COX-2 inhibition is effective. An independent-led, two-dimensional analysis of proportional reporting ratios (PRR) of individual NSAIDS against all drugs present within FAERS 2003-Q2 2017 was performed. Its unique feature of gaining insights on potentially beneficial effects in some cancers for ibuprofen and naproxen should warrant further research.
\end{abstract}

\section{Introduction}

Non-steroidal anti-inflammatory drugs (NSAIDs) are amongst the most widely used drugs worldwide to treat conditions were COX-2 inhibition is effective. Level 1 evidence has demonstrated the gastrointestinal toxicity of NSAIDs for decades. On the other hand, inflammation is considered a step in the cascade of events leading to the development of neoplasms. The FDA Adverse Event Reporting System (FAERS) is a database that contains adverse event reports, medication error reports and product quality complaints resulting in adverse events that were submitted to FDA (www.fda.gov). Our research question was: Are there differential reporting proportions of malignancies and gastrointestinal disorders between NSAIDs in the FDA Adverse Reporting System?

\section{Methods}

An independent-led, two-dimensional analysis of proportional reporting ratios (PRR) of individual NSAIDS against all drugs present within FAERS 2003-Q2 2017. Cut-off values were 0.5 for inferiority and 2 for superiority according to the criteria by Evans and colleagues established in 2001. GI and malignancies reports were identified by MedDRA SMQs 20000103 and 20000090, respectively. Target drugs were identified using the WHO anatomical therapeutic chemical classification (ATC) and included etodolac, tenoxicam, meloxicam, celecoxib, rofecoxib, nimesulide, diclofenac, indomethacin, aspirin, flurbiprofen, naproxoxen and ibuprofen. The PRR is a statistic that is used in disproportionality analyses to summarize the extent to which a particular adverse event is reported for individuals taking a specific drug, compared to the frequency at which the same adverse event is reported for patients not taking the drug. The cut-off used are 0.5 and 2 for inferiority and superiority, respectively [1].

\section{Results}

Insights 1: The vast majority of NSAIDS were associated with more GI and similar malignancies reports as compared to other drugs (rofecoxib, nimesulide, diclofenac, meloxicam, indomethacin, aspirin, ketoprofen, flurbiprofen).
Insights 2: celecoxib, tenoxicam and etodolac were associated with similar GI and malignancies reports as compared to other drugs

Insights 3: Ibuprofen and naproxen were associated with more reports of GI reports while less malignancies reports as compared to other drugs (graph 1).

\section{Discussion and conclusion}

Although not carrying the highest level of evidence, this analysis is insightful in reporting real-life safety profiles of currently marketed NSAIDs. Its limitations include underreporting, stimulated reporting, and Webber effect. Notwithstanding, its

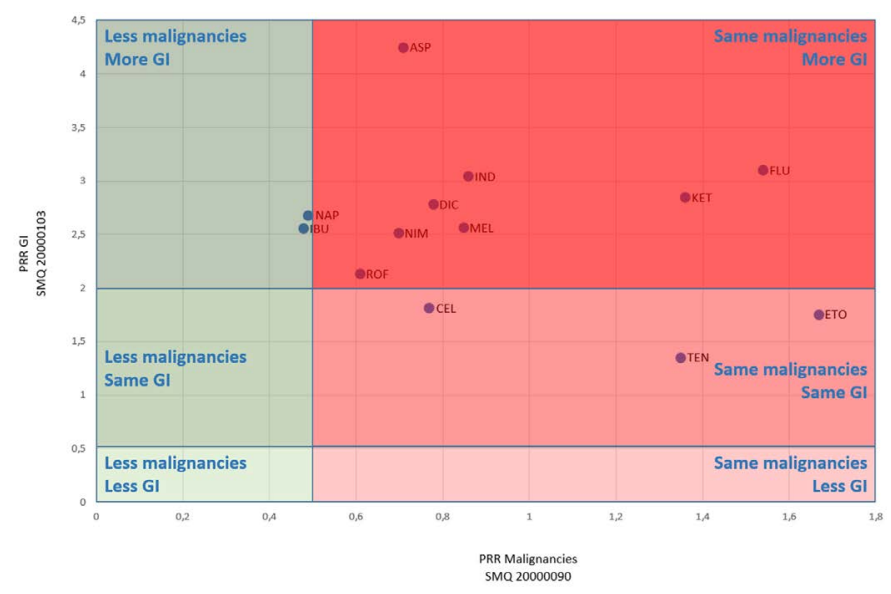

Graph 1. Two-dimensional mapping of NSAIDs with regards to reported gastrointestina disorders and malignancies

${ }^{\star}$ Correspondence to: Florent Richy, MBBS, MPH, PhD Director Bluepoint Consulting SCS, Liege, Belgium/Consultant professor University of Liege, Belgium, Email: bluepointconsultingscs@gmail.com

Received: June 14, 2018; Accepted: June 21, 2018; Published: June 25, 2018 
unique feature of gaining insights on potentially beneficial effects in some cancers for ibuprofen and naproxen should warrant further research.

\section{References}

1. Evans SJ, Waller PC, Davis S (2001) Use of proportional reporting ratios (PRRs) for signal generation from spontaneous adverse drug reaction reports. Pharmacoepidemiology and drug safety 10: 483-486. [Crossref]

Copyright: (C2018 Richy F. This is an open-access article distributed under the terms of the Creative Commons Attribution License, which permits unrestricted use, distribution, and reproduction in any medium, provided the original author and source are credited. 